\title{
Agricultural Pollution Risks Influence Microbial Ecology in Honghu Lake
}

2

3 Maozhen Han ${ }^{1,2,+}$, Melissa Dsouza ${ }^{3,4,5,+}$, Chunyu Zhou ${ }^{1,+}$, Hongjun $\mathrm{Li}^{1}$, Junqian Zhang ${ }^{6}$, Chaoyun Chen ${ }^{1}$, Qi Yao ${ }^{1}$, 4 Chaofang Zhong ${ }^{1}$, Hao Zhou ${ }^{1}$, Jack A Gilbert ${ }^{3,4,5, *}$, Zhi Wang ${ }^{2,}$, Kang Ning ${ }^{1, *}$

5

$6{ }^{1}$ Key Laboratory of Molecular Biophysics of the Ministry of Education, Hubei Key Laboratory of Bioinformatics

7 and Molecular-imaging, Department of Bioinformatics and Systems Biology, College of Life Science and

8 Technology, Huazhong University of Science and Technology, Wuhan, Hubei 430074, China

$9{ }^{2}$ Key Laboratory for Environment and Disaster Monitoring and Evaluation of Hubei, Institute of Geodesy and

10 Geophysics, Chinese Academy of Sciences, Wuhan, Hubei 430077, China

$11{ }^{3}$ The Microbiome Center, Department of Surgery, University of Chicago, Chicago, IL, 60637, USA

$12{ }^{4}$ Argonne National Laboratory, Biosciences Division, Lemont, IL, 60439, USA

$13{ }^{5}$ Marine Biological Laboratory, Woods Hole, MA, 02543, USA

$14{ }^{6}$ State Key Laboratory of Water Ecology and Biotechnology, Institute of Hydrobiology, Chinese Academy of 15 Sciences, Wuhan, Hubei 430072, China

16

17

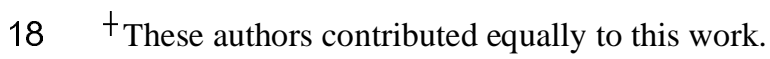

19 * Corresponding author. E-mail: ningkang@ @ust.edu.cn, zwang@ whigg.ac.cn, gilbertjack@ gmail.com 


\section{Abstract}

22 Background: Agricultural activities, such as stock-farming, planting industry, and fish aquaculture,

23 can influence the physicochemistry and biology of freshwater lakes. However, the extent to which

24 these agricultural activities, especially those that result in eutrophication and antibiotic pollution,

25 effect water and sediment-associated microbial ecology, remains unclear.

26 Methods: We performed a geospatial analysis of water and sediment associated microbial

27 community structure, as well as physicochemical parameters and antibiotic pollution, across 18 sites

28 in Honghu lake, which range from impacted to less-impacted by agricultural pollution. Furthermore,

29 the co-occurrence network of water and sediment were built and compared accorded to the

30 agricultural activities.

31 Results: Physicochemical properties including $\mathrm{TN}, \mathrm{TP}, \mathrm{NO}_{3}{ }^{-} \mathrm{N}$, and $\mathrm{NO}_{2}{ }^{-} \mathrm{N}$ were correlated with

32 microbial compositional differences in water samples. Likewise, in sediment samples, Sed-OM and

33 Sed-TN correlated with microbial diversity. Oxytetracycline and tetracycline concentration described

34 the majority of the variance in taxonomic and predicted functional diversity between impacted and

35 less-impacted sites in water and sediment samples, respectively. Finally, the structure of microbial

36 co-associations was influenced by the eutrophication and antibiotic pollution.

37 Conclusion: These analyses of the composition and structure of water and sediment microbial

38 communities in anthropologically-impacted lakes are imperative for effective environmental

39 pollution monitoring. Likewise, the exploration of the associations between environmental variables

40 (e.g. physicochemical properties, and antibiotics) and community structure is important in the

41 assessment of lake water quality and its ability to sustain agriculture. These results show agricultural

42 practices can negatively influence not only the physicochemical properties, but also the biodiversity 
43 of microbial communities associated with the Honghu lake ecosystem. And these results provide

44 compelling evidence that the microbial community can be used as a sentinel of eutrophication and

45 antibiotics pollution risk associated with agricultural activity; and that proper monitoring of this

46 environment is vital to maintain a sustainable environment in Honghu lake.

47 Keywords: freshwater, microbial communities, agriculture activities, antibiotics, human impact

48

\section{Background} 57 understudied.
Water ecosystems, especially inland lakes, have suffered from eutrophication associated with increased agricultural activity comprising fish aquaculture as well as crop and livestock farming on surrounding lands(Brooks et al. 2016, Geist and Hawkins 2016, Williams et al. 2016). Improperly managed agricultural activities, such as excessive and/or improper use of fertilizers and/or pesticides, can cause eutrophication, which can negatively impact biodiversity(Williams et al. 2004). Previous studies have focused on the impact of this pollution on macro-organismal communities(Verdonschot et al. 2011, Williams et al. 2004) and in comparison, microbial ecology remains relatively

Agricultural pollution alters the physicochemical properties of water ecosystems(Baquero et al. 2008), which changes the associated microbial community composition and structure. In particular, nitrogen and phosphorus content, water temperature, and $\mathrm{pH}$ can fundamentally influence the microbiome(Bowles et al. 2014, Lindström et al. 2005, Xu et al. 2010). However, few studies have quantified the impact of organic pollutants such as herbicides and antibiotics. Determining the ecosystems resilience to such disturbance can aid conservation and help in the development of remediation strategies. There is an urgent need to develop sustainable approaches that establish a 
balanced relationship between the environment and agricultural production.

67 for the prophylactic or curative treatment of infectious disease(Lee et al. 2007), yet surface runoff of

68 the introduction of treated sewage can introduce antibiotic pollution into local water bodies.

69 Antibiotics inhibit microbial activity and can therefore influence biogeochemical processes in these

70 ecosystems(Sengupta et al. 2013) and potentially select for antibiotic resistance mechanisms in

71 environmental bacteria(Cherkasov et al. 2008). In addition, animal sewage can introduce

72 animal-associated antibiotic resistant bacteria into these environments(Wang et al. 2017a), and as

73 such it is necessary to have better quantification of the fitness and recovery rates of these resistant

74 microbes upon release into the environment(Pei et al. 2006).

75 Honghu lake is a large, shallow eutrophic lake with an area of $\sim 350 \mathrm{~km}^{2}$ and an average depth

76 of $\sim 1.5 \mathrm{~m}$ (Figure 1). It is located between the irrigation channel of the Four-lake main canal and the

77 Yangzi River. Over the last five decades, Honghu lake has been extensively altered by flood

78 regulation, irrigation, fish aquaculture, shipping, and water supply demands(Ban et al. 2014, Zhang

79 1998). Today, more than $40 \%$ of the lake area is used for large-scale aquaculture(Zhang et al. 2017).

80 The intensive use of Honghu lake resources and the emission of sewage and other pollutants

81 including fertilizers, pesticides, and antibiotics into the lake have led to a severe degradation of its

82 water quality and an increase in the frequency of eutrophication events. In 2004, the Honghu Lake

83 Wetland Protection and Restoration Demonstration Project(Zhang et al. 2017) was implemented to

84 ameliorate the negative effects of severe water pollution, and one third of the lake area has been

85 gradually protected under this provision. Consequently, Honghu lake represents a valuable, natural

86 field site for investigating both the efficacy of the restoration program and the long-term effects of 
87 agricultural activities, such as the excessive application of antibiotics on water microbial

88 communities.

This study aimed to understand the geospatial influence of pollution on the water and

90 sediment-associated microbial communities in Honghu lake. We performed 16S rRNA amplicon

91 sequencing to characterize the microbial ecology, which was correlated against physicochemistry and

92 antibiotic concentrations in these environments. This research was guided by the following scientific

93 questions: (i) How does microbial diversity differ between water and sediment, and how is this

94 influenced by the intensity of agricultural pollution? (ii) Which physicochemical properties and

95 antibiotics are correlated with changes in microbial community structure? (iii) How are the

96 co-occurrence relationships between microbiota influenced by the intensity of agricultural pollution

97 (impacted and less-impacted)? Importantly, this baseline study aims to generate microbioal

98 biomarkers of pollution, and to identify the ecological trends that could be used to provide a sentinel

99 of pollution events in this lake environment.

100

101 Results

102 Physicochemical and antibiotic characterization

Physicochemical characteristics and antibiotic concentrations were determined for all water and sediment samples (Figure 1, Supplementary Tables S1, S2, and S4). Significant differences in pH

105 ( $t$-test, $P=0.0037), \mathrm{ORP}(t$-test, $P=0.00068), \mathrm{TN}$ ( $t$-test, $P=0.035)$, and $\mathrm{NH}_{4}{ }^{+}-\mathrm{N}(t$-test, $P=0.045)$

106 were observed between water samples from impacted and less-impacted (control) sites

107 (Supplementary Table S1). Samples from impacted sites were significantly more acidic and had

108 greater concentrations of ORP, $\mathrm{TN}$, and $\mathrm{NH}_{4}{ }^{+}-\mathrm{N}$ when compared to less-impacted sites 
109

110

111

112

113

114

115

116

117

118

119

120

121

122

123

124

125

126 127 observed in water samples.

128

129

130 were greater in impacted sites.

(Supplementary Table S1). Similarly, sediment samples maintained significantly different Sed-OM

( $t$-test, $P=0.002)$, Sed-LP $(t$-test, $P=0.0335)$, Sed-TN $(t$-test, $P=0.0013)$, and Sed-TP $(t$-test, $P=0.021)$

levels between impacted and less-impacted sites (Supplementary Table S2). Less-impacted

sediment had greater concentrations of Sed-OM, Sed-LP, and Sed-TN when compared to the

impacted sites (Supplementary Table S2), which may largely be due to the decomposition of plant

material over the preceding winter months. Between impacted and less-impacted sites, the antibiotics

ofloxacin (OFL, $t$-test, $P=0.0079$ ) and sulfamethoxazole (SMZ, $t$-test, $P=0.043$ ) had significantly

different concentrations in water samples, while sulfamerazine (SMR, $t$-test, $P=0.021$ ) was

significantly different in sediment samples (Supplementary Table S4); in both cases concentrations

\section{Microbial diversity and community structure}

A total of 28 water and sediment samples generated 4,441,405 paired-end 16S rRNA reads, which clustered into 7,785 OTUs (Supplementary Information). Microbial alpha diversity was significantly greater in sediment samples (Chao1 ( $t$-test, $P=0.0045$, Supplementary Table S5) and PD whole tree ( $t$-test, $P=0.003$, Supplementary Table S5)). The microbial alpha diversity in sediment samples was significantly different between impacted and less-impacted sites ( $t$-test, $P=0.0445$, Supplementary Table S5). However, no significant difference in alpha diversity was

A total of 53 microbial phyla were identified across all samples (Figure 2A), and were differentiated between water and sediment samples (Figure 2B), and between impacted and less-impacted sites (PERMANOVA, Bray-Curtis distance, $P<0.01$ ). In water samples, Proteobacteria 
131 ( $t$-test, $P<0.05)$, Cyanobacteria $(t$-test, $P<0.05)$, and Gemmatimonadetes $(t$-test, $P<0.05)$ were

132 significantly different between impacted and less-impacted sites (Figure 2C). While in sediment

133 samples, Actinobacteria ( $t$-test, $P<0.01)$, Firmicutes ( $t$-test, $P<0.05)$, Bacteroidetes $(t$-test, $P<0.05)$,

134 Nitrospirae $(t$-test, $P<0.05)$, and OP8 ( $t$-test, $P<0.05)$ were significantly different between impacted

135 and less-impacted sites (Figure 2D).

136 Core-OTUs were defined as a set of OTUs that were identified in all samples analyzed, and

137 Pan-OTUs were defined as a set of OTUs that were identified in at least one sample. Core- and

138 Pan-OTUs were determined for all water and sediment samples (Figure 3). A total of 132

139 Core-OTUs and 7,418 Pan-OTUs were identified in less-impacted sites, while impacted sites

140 maintained 201 Core-OTUs and 7,706 Pan-OTUs (Supplementary Figure S3 and Supplementary

141 Figure S4). The Core-OTUs from both the impacted and less-impacted sites were dominated by

142 Proteobacteria, specifically Janthinobacterium (Supplementary dataset 4 and 5), while

143 Acidobacteria were enriched at the impacted sites $(2.79 \% \pm 1.30 \%$, Supplementary dataset 4).

144 Microbial beta diversity was further assessed by UPGMA clustering using the unweighted

145 UniFrac distance matrix. We observed clustering by sampling medium (Figure 4A and

146 Supplementary Figure S5) and by level of agricultural activity within water and sediment samples

147 (Figure 4B). Importantly, greater differences in beta diversity were observed between impacted and

148 less-impacted sites in sediment samples as compared to water samples (Figure 4B and 4C).

\section{Functional properties predicted by PICRUSt}

We observed clustering of water and sediment microbial communities based on the relative 
153

154

155

156

157

158

Bray-Curtis distance, $P<0.0001)$. In water samples, functional groups including amino acid related enzymes, peptidases, oxidative phosphorylation, purine metabolism, pyrimidine metabolism, DNA repair and recombination proteins, and arginine and proline metabolism were enriched (Supplementary Figure S7). Likwise, in sediment samples we observed an enrichment of functional groups including ribosome biogenesis, secretion system, two-component system, ABC transporters, and pyruvate metaolism (Supplementary Figure S7). When investigating agricultural pollution risks, we observed significant differences in the relative abundances of the predicted functional profiles between impacted and less-impacted groups of water samples (PERMANOVA, Bray-Curtis distance, $P<0.05)$. For these samples, the relative abundances of DNA repair and recombination proteins ( $t$-test, $P<0.05)$, purine metabolism $(t$-test, $P<0.05)$, secretion systems $(t$-test, $P<0.05)$, oxidative phosphorylation ( $t$-test, $P<0.05$ ), pyrimidine metabolism ( $t$-test, $P<0.05$ ), amino acid related enzymes ( $t$-test, $P<0.05)$, and arginine and proline metabolism $(t$-test, $P<0.05)$ were significantly different between impacted and less-impacted sites (Supplementary Figure S7). In contrast, we observed no significant differences in sediment functional profiles between impacted and less-impacted sites.

\section{Correlating physicochemical properties with Microbial diversity}

Physicochemical properties including $\mathrm{NH}_{4}{ }^{+}-\mathrm{N}, \mathrm{TN}, \mathrm{ORP}, \mathrm{TP}$, Tur, $\mathrm{COD}_{\mathrm{Mn}}$, and Chl (Supplementary dataset 6) were significant explanatory factors that determined the observed clustering pattern of the water microbial communities at impacted sites (Figure 5A, Supplementary Figure S8A), while $\mathrm{pH}$ and $\mathrm{DO}$ determined the water microbial community structure at less-impacted sites (Figure 5A, Supplementary Figure S8A). For sediment samples, Sed-LP, 

shaping the observed clustering pattern at less-impacted sites and Sed-TP for impacted sites (Figure

177 5B, Supplementary Figure S8B). Based on distance correlations and the statistical significance of 178 Mantel's r-statistic, water physciochemical properties including $\mathrm{TN}, \mathrm{ORP}, \mathrm{NO}_{3}{ }^{-} \mathrm{-}$, and $\mathrm{NO}_{2}{ }^{-}-\mathrm{N}$, were strongly correlated with taxonomic and functional composition (Figure 6A). For sediment samples, Sed-OM and Sed-TN were strongly correlated with taxonomic data (Figure 6B).

The antibiotic oxytetracycline (OTC) was the primarily explanatory factor for water microbial diversity variance at impacted sites (Figure 5C, Supplementary Figure S8A). While in sediment samples, sulfamerazine (SMR) was the primary factor responsible for the observed clustering of samples, including R1S, R2S, P1S, P3S, and P4S, from impacted sites (Figure 5D, Supplementary Figure S8B). Mantel correlation assessments were also performed between antibiotic data and compositional data for water and sediment samples (Figure 6C, Figure 6D, and Supplementary Figure S9). The oxytetracycline (OTC) antibiotic class was strongly correlated with water taxonomic and functional composition data (Figure 6C), while tetracycline (TC) was strongly correlated with sediment taxonomic and functional composition data (Figure 6D). The ofloxacin (OFL) antibiotic class was strongly correlated with taxonomic and functional composition data in sediment samples collected from less-impacted (control) sites (Supplementary Figure S10B). Additionally, COD $\mathrm{Mn}_{\mathrm{n}}$ and ciprofloxacin (CIP) were strongly correlated with taxonomic and functional composition in water samples collected from impacted sites (Supplementary Figure S10C).

Moreover, we observed strong correlations between several OTUs, physicochemical properties, and antibiotic concentrations (Supplementary Information). In water samples, Bacillus flexus (denovo 71031, Supplementary dataset 8) was strongly correlated with TN ( $r=0.8675$, 
197

198

199

200

201

202

203

204

205

206

207

208

209

210

fdr- $p=7.89 \mathrm{E}-5$, Supplementary Figure S11C), $\mathrm{NH}_{4}{ }^{+} \mathrm{N}(r=0.8958$, fdr- $p=7.89 \mathrm{E}-5$, Supplementary

Figure S11D), $\mathrm{PO}_{4}{ }^{3-}-\mathrm{P}(r=0.832$, fdr- $p=2.58 \mathrm{E}-4$, Supplementary Figure S11E), and oxytetracycline (OTC, $r=0.8381$, fdr- $p=3.62 \mathrm{E}-4$, Supplementary Figure S12B).

\section{Biomarker discovery}

In water samples, the LEfSe analysis identified 13 biomarkers for impacted sites and 12 for less-impacted sites. The most differentially abundant bacteria from impacted sites belonged to the phylum Proteobacteria, class Betaproteobacteria and class Gammaproteobacteria (Figure 7A and 7B). These included members of the orders Methylophilales, Nitrosomonadales, and Rhodocyclales (Figure 7A and 7). Methylophilales are known for their ability to metabolize methane under aerobic and microaerobic conditions(Beck et al. 2013) and Nitrosomonadales are significantly enriched in soils containing high concentrations of $\mathrm{N}$ fertilizer(Chávez-Romero et al. 2016). Water samples from less-impacted sites were overrepresented by Oscillatoriophycideae and Synechococcophycideae in Cyanobacteria; and Saprospiraceae in Bacteroidetes (Figure 7A and 7B).

In sediment samples, the LEfSe analysis reported 14 biomarkers enriched in impacted sites and 5 enriched in less-impacted sites (Figure 7C and 7D). Biomarkers in samples from impacted sites mainly comprised members of the phylum Actinobacteria, family Pseudomonadaceae, order Burkholderiales, and class Flavobacteriia.. For sediment samples from less-impacted sites, bacteria that were differentially abundant include members of Paenisporosarcina genus and candidate family planococcaceae, phylum Firmicutes, order Bacillales, and class Bacilli (Figure 7 C and 7D).

\section{Co-occurrence Network Analysis}



low degree values and fewer hub nodes had high degree values (Supplementary Figure S15). members of Anaerolineae of the phylum Chloroflexi and Beta-, Delta-, and Gammaproteobacteria. observed fewer and smaller hubs in the water network. In this network, most OTUs in module 2 (22 nodes) and module 9 (12 nodes) were members of the genus Synechococcus within the order Synechococcales and ACK-M1 within the order Actinomycetales, respectively. 
241 OTUs associated with less-impacted samples as compared to those associated with samples from

242 impacted sites. This observation was confirmed when we generated sub-networks for impacted and

243 less-impacted sediment samples by selecting OTUs associated with these samples and all edges

244 among them from the overall sediment co-occurrence network (Supplementary Figure S16). We

245 observed higher connectedness in microbes associated with less-impacted samples (measured as

246 node degree, 3.746) as compared to those associated with samples from impacted sites (1.397).

248 Discussions

249 The extensive application of chemical compounds such as fertilizers, herbicides, and antibiotics,

250 can profoundly influence the cycling and accumulation of nutrients in the sediment and water

251 column of Honghu lake(Chen et al. 2008). These agricultural practices can negatively impact not

252 only the physicochemical properties, but also the biodiversity of microbial communities associated

253 with the lake ecosystem(Baquero et al. 2008). These changes in microbial community composition

254 can in turn affect nutrient cycling and organic matter decomposition, thus impacting overall

255 agricultural productivity.

256 In our study, we analyzed water and sediment samples from Honghu lake, assessing its

257 microbiome, physicochemical properties, and antibiotic concentrations. We found that despite low

258 human activity, high concentrations of Sed-LP, Sed-TN, and Sed-OM were observed at less-impacted

259 (control) sites, probably due to the abundance of submerged plants. We speculate that the decay of

260 these plants during winter subtantially increases organic matter, total nitrogen(Sand-jensen 1998),

261 and total phosphorus(Horppila and Nurminen 2003) in sediment samples. Hence, as expected from

262 previous research, we found that both water and sediment microbial community structure was 

samples, we observed that Bacillus flexus was strongly correlated with $\mathrm{TN}, \mathrm{NH}_{4}{ }^{+}-\mathrm{N}, \mathrm{PO}_{4}{ }^{3-}-\mathrm{P}$, and oxytetracycline. More important, previous work on Bacillus flexus have shown that members of this species can degrade organic(Guo et al. 2013) and inorganic(Divyashree et al. 2009) nitrogen, thus making it a possible candidate for bioremediation in alkaline wastewater(Wang and Zhao 2013). Some strains of B. flexus also demonstrate strong phosphorus solubilization activity(Gechemba et al. 2015), and others demonstrated resistance to OTC(Sundaramanickam et al. 2015). Hydrogenophaga genus, belonging to Burkholderiales (Class Betaproteobacteria), which have been

272 previously associated with agricultural activities(Babujia et al. 2016). Moreover, members of the genus Pseudomonas, belonging to family Pseudomonadaceae, can play an important role in

274 agricultural ecosystems, particularly those associated with plant growth-promotion and disease 275 suppression were mentioned(Pesaro and Widmer 2006). metabolize soluble organic substrates(Shao et al. 2013). Among these classes, Deltaproteobacteria, a 
processes and microbial competitive interactions within this ecosystem(Dennis et al. 2013). The

286 presence of these microbial taxa is indicative of the long-term effect of eutrophication in water

287 environments.

\section{Conclusion}

We analyzed the impacted sites and less-impacted sites of water and sediment samples from variable physicochemical properties and antibiotic concentrations. There were significant differences between impacted and less-impacted (control) groups in both water and sediment samples. These differences were observed in physicochemical properties, antibiotic concentration levels, and taxonomic structure. Physicochemical properties including TN, TP, NO3--N, and NO2--N were the main factors driving compositional differences in water samples. Likewise, in sediment samples,

297 Sed-OM and Sed-TN were the main factors driving differences in taxonomic composition. The antibiotics, oxytetracycline and tetracycline were identified as the main drivers of taxonomic and

299 functional structure in water and sediment samples, respectively. As for differences between

300 impacted and less-impacted samples, we identified 25 biomarkers within water communities and 19

301 within sediment microbial communities. Finally, the co-occurrence network analysis revealed 302 differences in co-occurrence patterns by sampling medium (water vs. sediment microbial 303 communities) and by level of agricultural activity (impacted vs. less-impacted microbial 304 communities). These results suggest that continued analyses of the composition and structure of water and sediment microbial communities in such anthropologically-impacted lake environments may provide valuable biomarker data to track pollution. The Honghu Lake Wetland Protection and 
307 Restoration Demonstration Project provided preliminary data that highlights the importance of

308 monitoring biodiversity in water micro-ecosystems. Our present work allows further investigation

309 into the impact of agricultural practices on water ecosystems and more importantly, into our ability to

310 remediate these important ecosystems.

312 Materials and Methods

313 Sample sites and sampling processes

314 To investigate the differences in microbial community structure resulting from a wide range of 315 anthropogenic activities, a total of 14 water samples and 14 sediment samples were collected from 316 Honghu lake and surrounding rivers and ponds during 10-11 November 2015. Among these sites, L1

317 is the inlet of inflowing river, and sites L3, L8, L9, and L10 are relatively close to aquaculture areas.

318 Meanwhile, to evaluate the primary source of the antibiotics of Honghu lake, the waters from four 319 major connecting rivers (i.e., R1 to R4) of Honghu lake and four typical aquaculture ponds (i.e., P1 to P4), which can exchange water with Honghu lake, were collected(Wang et al. 2017b). In keeping with the Government Protection Zone definition(Zhang et al. 2017) and in taking into account the different sources of pollution at each site(Wang et al. 2017b) (treated sewage, crop, livestock, and less-impacted, control group(Zhang et al. 2017). Sampling sites labeled L1, L2, P1, P2, P3, P4, R1, were classified as less-impacted (Figure 1). 

Microbial biomass was collected on $0.22 \mu \mathrm{m}$ diameter pore size filter membranes. These membrane samples were stored onsite in a portable cooler with ice bags, then transported to the laboratory and stored at $-80^{\circ} \mathrm{C}$ until DNA extraction. For sediment sampling, $\sim 200 \mathrm{~g}$ of sediment $(0-10 \mathrm{~cm})$ was collected at each site and stored in a portable cooler with ice bags until its transportation to the laboratory for subsequent downstream analyses. Approximately $50 \mathrm{~g}$ of sediment was used for physicochemical characterization and antibiotic analysis, whilst the remainder was dried in an Ultra-low Freeze Dryer (Christ, German) until no further weight changes were observed. The dried sediment $(0.5 \mathrm{~g})$ was used for DNA extraction.

\section{Physicochemical characterization and antibiotic analysis}

347 phosphorus (TP), orthophosphate $\left(\mathrm{PO}_{4}{ }^{3-}-\mathrm{P}\right)$, total nitrogen $(\mathrm{TN})$, ammonium nitrogen $\left(\mathrm{NH}_{4}{ }^{+}-\mathrm{N}\right)$,

348 nitrate nitrogen $\left(\mathrm{NO}_{3}{ }^{-} \mathrm{N}\right)$, nitrite nitrogen $\left(\mathrm{NO}_{2}{ }^{-} \mathrm{N}\right)$, and potassium permanganate index (oxygen 349 consumption, $\mathrm{COD}_{\mathrm{Mn}}$ ) were assayed as described in previous work (Federation and Association 350 2005). For sediment samples, ORP (Sed-ORP) and $\mathrm{pH}$ (Sed-pH) were determined using a pH/ORP 
351 portable meter (YSI, USA). Sediment organic matter (Sed-OM) was determined in a muffle furnace

352 at $550^{\circ} \mathrm{C}\left(\right.$ Federation and Association 2005). Labile phosphorus (LP), $\mathrm{NH}_{4}{ }^{+} \mathrm{N}$ (Sed- $\left.\mathrm{NH}_{4}{ }^{+}-\mathrm{N}\right)$, total

353 phosphorus (Sed-TP), and total nitrogen (Sed-TN) were measured by the $\mathrm{NH}_{4} \mathrm{Cl}$ extraction method,

354 the $\mathrm{KCl}$ extraction method, the perchloric acid and sulfuric acid digestion method, and the Kjeldahl

355 method, respectively(Bao 2005).

356 Antibiotic analysis: Based on a report of antibiotic usage in China(Yang et al. 2016), a total of

35713 antibiotics were selected for detection in water and sediment samples (Supplementary Table S4).

358 These antibiotics can be classified into three groups namely: (i) sulfonamides (SAs), including

359 sulfadiazine (SDZ), sulfamerazine (SMR), sulfamate (SFM), sulfadimidine (SMD),

360 sulfamonomethoxine (SMM), and sulfamethoxazole (SMZ); (ii) fluoroquinolones (FQs), including

361 fleroxacin (FLE), ofloxacin (OFL), ciprofloxacin (CIP), and difloxacin (DIF); and (iii) the

362 tetracycline group (TCs), including tetracycline (TC), oxytetracycline (OTC), and chlortetracycline

363 (CTC). We determined the concentration of these antibiotics in water and sediment samples using a

3642695 Waters Alliance system (Milford, MA, USA) equipped with an auto sampler-controlled binary

365 gradient system, a micro vacuum degasser, and a 2998 Photodiode Array (PDA) detector. A detailed

366 protocol of the antibiotic extraction process is described in Supplementary Information. Of the 13

367 antibiotics that were quantified, nine antibiotics including TC, OTC, CTC, SDZ, SMR, SMD, OFL,

368 CIP, and SMZ were selected for further analysis in this study.

\section{DNA extraction and $16 \mathrm{~S}$ rRNA gene sequencing}


374 and stored at $-20^{\circ} \mathrm{C}$ until further use.

DNA samples were quantified using a Qubit $^{\circledR} 2.0$ Fluorometer (Invitrogen, Carlsbad, CA) and

376 DNA quality was assessed on $0.8 \%$ agarose gels. Approximately 5-50 ng of DNA was used as

377 template for amplifying the V4-V5 hypervariable region of the 16S rRNA gene of microbiota for 378 each sample. Sequences for the forward and reverse primers are "GTGYCAGCMGCCGCGGTAA"

379 and "CTTGTGCGGKCCCCCGYCAATTC", respectively(Han et al. 2016). The sequencing library 380 was constructed using a MetaVx ${ }^{\mathrm{TM}}$ Library Preparation kit (GENEWIZ, Inc., South Plainfield, and 381 NJ, USA). Indexed adapters were added to the ends of the 16S rDNA amplicons by limited cycle

PCR. DNA libraries were verified using an Agilent 2100 Bioanalyzer (Agilent Technologies, Palo

383 Alto, CA, USA) and quantified by Qubit ${ }^{\circledR} 2.0$ and quantitative PCR (Applied Biosystems, Carlsbad,

384 CA, USA). All sequencing reactions were performed on the Illumina MiSeq platform using 385 paired-end sequencing technology $(2 * 300 \mathrm{bp})$.

\section{Quality control, OTU clustering, and taxonomy assignment}

All 16S rRNA gene amplicons were processed according to the ensuing criteria and sequences below the set quality threshold were excluded from subsequent analyses. Firstly, paired-end reads were spliced using the 'make.contigs' command in mothur(Schloss et al. 2009) (version 1.25.0) with default settings. All reads containing ambiguous base calls $(\mathrm{N})$, those longer than $500 \mathrm{bp}$, and those

392 shorter than 300 bp were removed. Putative chimeras were identified using the SILVA database(Quast et al. 2013) (Release 123) and removed using the 'chimera.uchime' and 'remove.seqs' commands in mothur. All high-quality sequences were aligned using PyNAST and 
396 Ecology, v1.9.1)(Caporaso et al. 2010b). Finally, the Greengenes database (version 13_8)(DeSantis

397 et al. 2006) was used as the reference database for classifying de novo operational taxonomic units

398 (OTUs) that were clustered at the 97\% nucleotide identity threshold. We set $0.001 \%$ as the threshold

399 to filter the low-abundance OTUs and keep abundant OTUs, i.e., only OTUs with the read

400 counts $>0.001 \%$ of the total reads of all samples were kept for analysis(Li et al. 2015).

401

402

\section{Microbial diversity assessment}

Microbial alpha- and beta-diversity values were determined using the QIIME(Caporaso et al. 2010b) pipeline. For alpha-diversity, rarefaction curves were drawn based on the following metrics:

40761,088 reads per sample. Bray-Curtis, weighted and unweighted UniFrac distance metrics(Lozupone

408 and Knight 2005) were used to measure community similarity between samples. Microbial

409 community clustering was arrayed by Principle Coordinate Analysis (PCoA) and visualized using

410 Emperor(Vázquez-Baeza et al. 2013) in QIIME. The hierarchical clustering method, UPGMA

411 (Unweighted Pair Group Method with Arithmetic Mean), was applied to cluster all water and

412 sediment samples, and the clustering tree was visualized in FigTree (version 1.4.2)(Rambaut 2014).

413 Permutational multivariate analysis of variance (PERMANOVA)(Anderson 2001) was performed on

414 the Bray-Curtis distance matrix to compare differences in community structure.

\section{Functional profiling}

417 PICRUSt (version 1.0.0-dev)(Langille et al. 2013) was used to make functional predictions 
418 based on the $16 \mathrm{~S}$ rDNA dataset from each sample. For this, OTU-picking was performed on all

419 quality-filtered sequence data using the 'pick_closed_reference_otus.py' command in QIIME. OTUs

420 were clustered at the $97 \%$ nucleotide identity threshold using the Greengenes database. The OTU

421 table was normalized using the 'normalize_by_copy_number.py' command. The normalized OTU

422 table was used for functional prediction with the 'predict_metagenomes.py' script, and functional

423 trait abundances were determined for each sample using the KEGG database (version 66.1, May 1,

424 2013)(Kang et al. 2016). Finally, the predicted functional content was collapsed to level three of the

425 KEGG hierarchy using the 'categorize_by_function.py' script.

427 Analysis of the relationships between physicochemical properties, antibiotics, and

microbial communities

Canonical correspondence analysis (CCA) was used to identify an environmental basis for community ordination, revealing relationships between microbial communities and environmental significant correlations between physicochemical properties, antibiotics, and microbial communities. properties and antibiotics) and OTUs, we applied a low-abundance filter to remove OTUs whose relative abundance did not exceed $0.01 \%$ in any sample (as previously reported by (Sunagawa et al. 2015)). Similarly, for physicochemical data and antibiotics data, the values of each variable were 
440 interactions between an environmental factor and an OTU, the threshold of the r-value and the False

441 Discovery Rate (FDR)-corrected-p-value of the Pearson Correlation Coeffcient was set at 0.8 and

4420.05 , respectively.

\section{Analysis of environmental drivers of microbial community composition}

445 We noted environmental drivers of microbial community composition on the basis of (i) 446 compositional data, which includes taxonomic composition (relative taxonomic abundances) and

447 functional composition at KEGG module level three; (ii) physicochemical data; and (iii) antibiotics

448 data. To pre-process compositional data, we applied a low-abundance filter to remove OTUs whose

449 relative abundance did not exceed $0.01 \%$ in any sample and then log transformed the relative

450 abundances. Likewise, for physicochemical and antibiotics data, the values of each variable were

451 transformed to $z$-scores. Based on the Euclidean distances, we computed Mantel correlations

452 between the physicochemical data and compositional data and then the antibiotics data and

453 compositional data (9,999 permutations). We obtained the results in $\mathrm{R}$ (version 3.3.1) and visualized

454 it in the Adobe Illustrator (version 16.0.0). Taxonomic composition and functional composition data

455 were correlated to each antibiotic and physicochemical property by Mantel's tests. The distance

456 correlations and the statistical significance of Mantel's r statistic corresponded to edge width and

457 edge color, respectively(Sunagawa et al. 2015).

458

459 Biomarker analysis

Based on their location, all water and sediment samples can be divided into two groups-impacted and less-impacted (control) groups. It is well known that the taxonomic 
462

463

464

465

466

467

composition of a microbial community can be impacted by local environmental variables. As a result, some bacteria might be enriched by distinctive environmental states. Linear discriminate analysis (LDA) effect size (LEfSe)(Segata et al. 2011) was used to select biomarkers in impacted and less-impacted (control) groups in water and sediment samples. Briefly, the taxa abundance table was imported into the LEfSe pipeline, and the parameters were set as follows: the alpha value for the factorial Kruskal-Wallis test(Breslow 1970) among classes and the $p$-value for the pairwise Wilcoxon test between subclasses were both chosen to be 0.05 . The threshold for the logarithmic LDA score for discriminative features was set at 3.0 and 3.5 for water and sediment samples, respectively.

\section{Co-occurrence network analysis}

To reduce sparsity, we selected water and sediment OTUs that were present in at least $50 \%$ of all water and sediment samples, respectively. We then generated separate networks for water and sediment microbial communities. The co-occurrence network was constructed using the CAVNet package(Cardona 2017) in $\mathrm{R}$ (as previously described by(Ma et al. 2016)). Briefly, water and sediment networks were inferred using the Spearman correlation matrix with the WGCNA package(Langfelder and Horvath 2012). In this network, co-occurring OTUs are represented by nodes and connected by edges. The network deconvolution method was utilized to distinguish direct correlation dependencies(Feizi et al. 2013). All p-values were corrected for multiple testing using the Benjamini and Hochberg FDR-controlling procedure(Benjamini et al. 2006). The cutoff of the FDR-corrected- $p$-value was set at 0.01. Random matrix theory-based methods were utilized to determine the cutoff of Spearman correlation coefficients for water (0.84) and sediment (0.81) networks. All network properties were calculated using the igraph package in $\mathrm{R}$ (Csardi and Nepusz 
less-impacted (control) sites using the 'plot_network_by_continuous_variable' function in CAVNet.

\section{References}

491 Anderson, M.J. (2001) A new method for non-parametric multivariate analysis of variance. Austral Ecology 26(1), $492 \quad 32-46$.

493 Babujia, L.C., Silva, A.P., Nakatani, A.S., Cantão, M.E., Vasconcelos, A.T.R., Visentainer, J.V. and Hungria, M. (2016) Impact of long-term cropping of glyphosate-resistant transgenic soybean [Glycine max (L.) Merr.] on soil microbiome. Transgenic Research, 1-16.

496 Ban, X., Wu, Q., Pan, B., Du, Y. and Feng, Q. (2014) Application of Composite Water Quality Identification Index on the water quality evaluation in spatial and temporal variations: a case study in Honghu Lake, China. Environmental monitoring and assessment 186(7), 4237-4247.

499 Bao, S. (2005) Agricultural and chemistry analysis of soil. Agric. Press, Beijing, China.

500 Baquero, F., Martínez, J.-L. and Cantón, R. (2008) Antibiotics and antibiotic resistance in water environments.

501 Current Opinion In Biotechnology 19(3), 260-265.

502 Beck, D.A., Kalyuzhnaya, M.G., Malfatti, S., Tringe, S.G., del Rio, T.G., Ivanova, N., Lidstrom, M.E. and 503 Chistoserdova, L. (2013) A metagenomic insight into freshwater methane-utilizing communities and evidence for 504 cooperation between the Methylococcaceae and the Methylophilaceae. PeerJ 1, e23.

505 Benjamini, Y., Krieger, A.M. and Yekutieli, D. (2006) Adaptive linear step-up procedures that control the false 506 discovery rate. Biometrika, 491-507.

507 Bowles, T.M., Acosta-Martínez, V., Calderón, F. and Jackson, L.E. (2014) Soil enzyme activities, microbial 508 communities, and carbon and nitrogen availability in organic agroecosystems across an intensively-managed 509 agricultural landscape. Soil Biology and Biochemistry 68, 252-262.

510 Breslow, N. (1970) A generalized Kruskal-Wallis test for comparing K samples subject to unequal patterns of 511 censorship. Biometrika 57(3), 579-594.

512 Brooks, B.W., Lazorchak, J.M., Howard, M.D., Johnson, M.V.V., Morton, S.L., Perkins, D.A., Reavie, E.D., Scott,

513 G.I., Smith, S.A. and Steevens, J.A. (2016) Are harmful algal blooms becoming the greatest inland water quality

514 threat to public health and aquatic ecosystems? Environmental Toxicology and Chemistry 35(1), 6-13.

515 Caporaso, J.G., Bittinger, K., Bushman, F.D., DeSantis, T.Z., Andersen, G.L. and Knight, R. (2010a) PyNAST: a 516 flexible tool for aligning sequences to a template alignment. Bioinformatics 26(2), 266-267.

517 Caporaso, J.G., Kuczynski, J., Stombaugh, J., Bittinger, K., Bushman, F.D., Costello, E.K., Fierer, N., Pena, A.G., 518 Goodrich, J.K. and Gordon, J.I. (2010b) QIIME allows analysis of high-throughput community sequencing data. 519 Nature Methods 7(5), 335-336.

520 Cardona, C. (2017) CAVNet. 
Chávez-Romero, Y., Navarro-Noya, Y.E., Reynoso-Martínez, S.C., Sarria-Guzmán, Y., Govaerts, B., Verhulst, N., Dendooven, L. and Luna-Guido, M. (2016) 16S metagenomics reveals changes in the soil bacterial community driven by soil organic $\mathrm{C}, \mathrm{N}$-fertilizer and tillage-crop residue management. Soil and Tillage Research 159, 1-8. Chen, M., Chen, J. and Sun, F. (2008) Agricultural phosphorus flow and its environmental impacts in China. Science of The Total Environment 405(1), 140-152.

Cheng, X., Chen, X., Su, X., Zhao, H., Han, M., Bo, C., Xu, J., Bai, H. and Ning, K. (2014a) DNA extraction protocol for biological ingredient analysis of LiuWei DiHuang Wan. Genomics, Proteomics \& Bioinformatics 12(3), 137-143.

Cheng, X., Su, X., Chen, X., Zhao, H., Bo, C., Xu, J., Bai, H. and Ning, K. (2014b) Biological ingredient analysis of traditional Chinese medicine preparation based on high-throughput sequencing: the story for Liuwei Dihuang Wan. Scientific Reports 4.

Cherkasov, A., Hilpert, K., Jenssen, H., Fjell, C.D., Waldbrook, M., Mullaly, S.C., Volkmer, R. and Hancock, R.E. (2008) Use of artificial intelligence in the design of small peptide antibiotics effective against a broad spectrum of highly antibiotic-resistant superbugs. ACS chemical biology 4(1), 65-74.

Crocker, L. and Algina, J. (1986) Introduction to classical and modern test theory, ERIC.

Csardi, G. and Nepusz, T. (2006) The igraph software package for complex network research. InterJournal, Complex Systems 1695(5), 1-9.

Dawson, K.S., Strapoć, D., Huizinga, B., Lidstrom, U., Ashby, M. and Macalady, J.L. (2012) Quantitative fluorescence in situ hybridization analysis of microbial consortia from a biogenic gas field in Alaska's Cook Inlet Basin. Applied and Environmental Microbiology 78(10), 3599-3605.

Dennis, P.G., Seymour, J., Kumbun, K. and Tyson, G.W. (2013) Diverse populations of lake water bacteria exhibit chemotaxis towards inorganic nutrients. The ISME Journal 7(8), 1661-1664.

DeSantis, T.Z., Hugenholtz, P., Larsen, N., Rojas, M., Brodie, E.L., Keller, K., Huber, T., Dalevi, D., Hu, P. and Andersen, G.L. (2006) Greengenes, a chimera-checked 16S rRNA gene database and workbench compatible with ARB. Applied and Environmental Microbiology. 72(7), 5069-5072.

Divyashree, M.S., Rastogi, N.K. and Shamala, T.R. (2009) A simple kinetic model for growth and biosynthesis of polyhydroxyalkanoate in Bacillus flexus. New Biotechnology 26(1), 92-98.

Federation, W.E. and Association, A.P.H. (2005) Standard methods for the examination of water and wastewater. American Public Health Association (APHA): Washington, DC, USA.

Feizi, S., Marbach, D., Médard, M. and Kellis, M. (2013) Network deconvolution as a general method to distinguish direct dependencies in networks. Nature biotechnology 31(8), 726-733.

Gechemba, O.R., Budambula, N., Makonde, H.M., Julius, M. and Matiru, V.N. (2015) Potentially beneficial rhizobacteria associated with banana plants in Juja, Kenya. Journal of Biodiversity and Environmental Sciences $7(2), 181-188$.

Geist, J. and Hawkins, S.J. (2016) Habitat recovery and restoration in aquatic ecosystems: current progress and future challenges. Aquatic Conservation: Marine and Freshwater Ecosystems.

Guo, D., Guan, L., Zhang, C., Wang, X. and Shan, L. (2013) UV Mutagenesis Breeding of Bacillus flexus Highly Degrading Organic Nitrogen. Guizhou Agricultural Sciences 11, 028.

Han, M., Gong, Y., Zhou, C., Zhang, J., Wang, Z. and Ning, K. (2016) Comparison and Interpretation of Taxonomical Structure of Bacterial Communities in Two Types of Lakes on Yun-Gui plateau of China. Sci Rep 6. Horppila, J. and Nurminen, L. (2003) Effects of submerged macrophytes on sediment resuspension and internal phosphorus loading in Lake Hiidenvesi (southern Finland). Water research 37(18), 4468-4474.

Kang, C., Zhang, Y., Zhu, X., Liu, K., Wang, X., Chen, M., Wang, J., Chen, H., Hui, S. and Huang, L. (2016) Healthy subjects differentially respond to dietary capsaicin correlating with specific gut enterotypes. The Journal of 
Clinical Endocrinology and Metabolism. 101(12), 4681-4689.

Langfelder, P. and Horvath, S. (2012) Fast R functions for robust correlations and hierarchical clustering. Journal of statistical software 46(11).

Langille, M.G., Zaneveld, J., Caporaso, J.G., McDonald, D., Knights, D., Reyes, J.A., Clemente, J.C., Burkepile, D.E., Thurber, R.L.V. and Knight, R. (2013) Predictive functional profiling of microbial communities using $16 \mathrm{~S}$ rRNA marker gene sequences. Nature Biotechnology. 31(9), 814-821.

Lee, L.S., Carmosini, N., Sassman, S.A., Dion, H.M. and Sepulveda, M.S. (2007) Agricultural contributions of antimicrobials and hormones on soil and water quality. Advances In Agronomy 93, 1-68.

Li, J., Zhang, J., Liu, L., Fan, Y., Li, L., Yang, Y., Lu, Z. and Zhang, X. (2015) Annual periodicity in planktonic bacterial and archaeal community composition of eutrophic Lake Taihu. Scientific Reports 5.

Lindström, E.S., Kamst-Van Agterveld, M.P. and Zwart, G. (2005) Distribution of typical freshwater bacterial groups is associated with $\mathrm{pH}$, temperature, and lake water retention time. Applied and Environmental Microbiology 71(12), 8201-8206.

Lozupone, C. and Knight, R. (2005) UniFrac: a new phylogenetic method for comparing microbial communities. Applied and Environmental Microbiology. 71(12), 8228-8235.

Ma, B., Wang, H., Dsouza, M., Lou, J., He, Y., Dai, Z., Brookes, P.C., Xu, J. and Gilbert, J.A. (2016) Geographic patterns of co-occurrence network topological features for soil microbiota at continental scale in eastern China. The ISME Journal.

Magurran, A.E. (2013) Measuring biological diversity, John Wiley \& Sons.

Nielsen, P.H., Kragelund, C., Seviour, R.J. and Nielsen, J.L. (2009) Identity and ecophysiology of filamentous bacteria in activated sludge. FEMS microbiology reviews 33(6), 969-998.

O'neil, J., Davis, T.W., Burford, M.A. and Gobler, C. (2012) The rise of harmful cyanobacteria blooms: the potential roles of eutrophication and climate change. Harmful Algae 14, 313-334.

Pei, R., Kim, S.-C., Carlson, K.H. and Pruden, A. (2006) Effect of river landscape on the sediment concentrations of antibiotics and corresponding antibiotic resistance genes (ARG). Water research 40(12), 2427-2435.

Pesaro, M. and Widmer, F. (2006) Identification and specific detection of a novel Pseudomonadaceae cluster associated with soils from winter wheat plots of a long-term agricultural field experiment. Applied And Environmental Microbiology 72(1), 37-43.

Pons, P. and Latapy, M. (2005) Computing communities in large networks using random walks. International Symposium on Computer and Information Sciences, 284-293.

Porebski, S., Bailey, L.G. and Baum, B.R. (1997) Modification of a CTAB DNA extraction protocol for plants containing high polysaccharide and polyphenol components. Plant molecular biology reporter 15(1), 8-15.

Quast, C., Pruesse, E., Yilmaz, P., Gerken, J., Schweer, T., Yarza, P., Peplies, J. and Glöckner, F.O. (2013) The SILVA ribosomal RNA gene database project: improved data processing and web-based tools. Nucleic Acids Research. 41(D1), D590-D596.

Rambaut, A. (2014) FigTree v1. 4.2. Edinburgh: University of Edinburgh.

Sand-jensen, K. (1998) Influence of submerged macrophytes on sediment composition and near-bed flow in lowland streams. Freshwater biology 39(4), 663-679.

Schloss, P.D., Westcott, S.L., Ryabin, T., Hall, J.R., Hartmann, M., Hollister, E.B., Lesniewski, R.A., Oakley, B.B., Parks, D.H. and Robinson, C.J. (2009) Introducing mothur: open-source, platform-independent, community-supported software for describing and comparing microbial communities. Applied and Environmental Microbiology. 75(23), 7537-7541.

Segata, N., Izard, J., Waldron, L., Gevers, D., Miropolsky, L., Garrett, W.S. and Huttenhower, C. (2011) Metagenomic biomarker discovery and explanation. Genome Biology 12(6), R60. 
Sengupta, S., Chattopadhyay, M.K. and Grossart, H.-P. (2013) The multifaceted roles of antibiotics and antibiotic resistance in nature. Frontiers in Microbiology 4.

Shao, K., Gao, G., Wang, Y., Tang, X. and Qin, B. (2013) Vertical diversity of sediment bacterial communities in two different trophic states of the eutrophic Lake Taihu, China. Journal of Environmental Sciences. 25(6), 1186-1194.

Sunagawa, S., Coelho, L.P., Chaffron, S., Kultima, J.R., Labadie, K., Salazar, G., Djahanschiri, B., Zeller, G., Mende, D.R. and Alberti, A. (2015) Structure and function of the global ocean microbiome. Science 348(6237), 1261359.

Sundaramanickam, A., Kumar, P.S., Kumaresan, S. and Balasubramanian, T. (2015) Isolation and molecular characterization of multidrug-resistant halophilic bacteria from shrimp farm effluents of Parangipettai coastal waters. Environmental Science and Pollution Research 22(15), 11700-11707.

Takamura N, K.Y., Fukushima M, Nakagawa M, KIM, B. H. (2003) Effects of aquatic macrophytes on water quality and phytoplankton communities in shallow lakes. Ecological Research 18(4), 381-395.

Ter Braak, C.J. (1986) Canonical correspondence analysis: a new eigenvector technique for multivariate direct gradient analysis. Ecology 67(5), 1167-1179.

Vázquez-Baeza, Y., Pirrung, M., Gonzalez, A. and Knight, R. (2013) EMPeror: a tool for visualizing high-throughput microbial community data. Gigascience 2(1), 1.

Verdonschot, R., Keizer-vlek, H.E. and Verdonschot, P.F. (2011) Biodiversity value of agricultural drainage ditches: a comparative analysis of the aquatic invertebrate fauna of ditches and small lakes. Aquatic Conservation: Marine and Freshwater Ecosystems 21(7), 715-727.

Virtanen, R., Ilmonen, J., Paasivirta, L. and Muotka, T. (2009) Community concordance between bryophyte and insect assemblages in boreal springs: a broad-scale study in isolated habitats. Freshwater Biology 54(8), 1651-1662. Wang, H., Sangwan, N., Li, H.-Y., Su, J.-Q., Oyang, W.-Y., Zhang, Z.-J., Gilbert, J.A., Zhu, Y.-G., Ping, F. and Zhang, H.-L. (2017a) The antibiotic resistome of swine manure is significantly altered by association with the Musca domestica larvae gut microbiome. The ISME Journal 11(1), 100-111.

Wang, X. and Zhao, H. (2013) Isolation and Characterization of a Bacillus flexus Strain Used in Alkaline Wastewater Treatment. Advanced Materials Research 750, 1381-1384.

Wang, Z., Du, Y., Yang, C., Liu, X., Zhang, J., Li, E., Zhang, Q. and Wang, X. (2017b) Occurrence and ecological hazard assessment of selected antibiotics in the surface waters in and around Lake Honghu, China. Science of The Total Environment 609(Supplement C), 1423-1432.

Williams, C.J., Frost, P.C., Morales-Williams, A.M., Larson, J.H., Richardson, W.B., Chiandet, A.S. and Xenopoulos, M.A. (2016) Human activities cause distinct dissolved organic matter composition across freshwater ecosystems. Global change biology 22(2), 613-626.

Williams, P., Whitfield, M., Biggs, J., Bray, S., Fox, G., Nicolet, P. and Sear, D. (2004) Comparative biodiversity of rivers, streams, ditches and ponds in an agricultural landscape in Southern England. Biological conservation 115(2), 329-341.

Wood, S.A., Maier, M.Y., Puddick, J., Pochon, X., Zaiko, A., Dietrich, D.R. and Hamilton, D.P. (2017) Trophic state and geographic gradients influence planktonic cyanobacterial diversity and distribution in New Zealand lakes. FEMS Microbiology Ecology 93(2), fiw234.

Xu, H., Paerl, H.W., Qin, B., Zhu, G. and Gao, G. (2010) Nitrogen and phosphorus inputs control phytoplankton growth in eutrophic Lake Taihu, China. Limnology and Oceanography 55(1), 420.

Yang, Y., Cao, X., Lin, H. and Wang, J. (2016) Antibiotics and Antibiotic Resistance Genes in Sediment of Honghu Lake and East Dongting Lake, China. Microbial Ecology 72(4), 791-801.

Ye, W., Liu, X., Lin, S., Tan, J., Pan, J., Li, D. and Yang, H. (2009) The vertical distribution of bacterial and 
653 archaeal communities in the water and sediment of Lake Taihu. FEMS Microbiology Ecology 70(2), 263-276.

654 Zhang, T., Ban, X., Wang, X., Cai, X., Li, E., Wang, Z., Yang, C., Zhang, Q. and Lu, X. (2017) Analysis of nutrient

655 transport and ecological response in Honghu Lake, China by using a mathematical model. Science of The Total

656 Environment 575, 418-428.

657 Zhang, X. (1998) On the estimation of biomass of submerged vegetation using Landsat thematic mapper (TM)

658 imagery: a case study of the Honghu Lake, PR China. International Journal Of Remote Sensing 19(1), 11-20.

659

\section{Declarations}

\section{Authors' contributions}

662 The whole study was designed by ZW and KN. MZH, JQZ and ZW collected samples. MZH,

663 CYC, QY and HZ performed DNA extraction and sequencing. MZH, CYZ, MD and HJL analyzed

664 the data. MZH, CYZ, MD, HJL, JG, ZW, and KN wrote the initial draft of the manuscript. All revised

665 the manuscript.

666 Funding

667 This work was partially supported by National Science Foundation of China grant 61103167,

66831271410 and 31671374, Ministry of Science and Technology's high-tech (863) grant 669 2012AA023107 and 2014AA021502, Key Project of Hubei Province Natural Science Foundation 670 (2015CFA132), Fundamental Research Funds for the Central Universities and Sino-German

671 Research Center grant GZ878.

672 Availability of data and material

673 All sequencing data for the 14 water samples and the 14 sediment samples were deposited into

674 NCBI's Sequence Read Archive (SRA) database under the Bioproject number PRJNA352457.

675 Competing financial interests

676 The authors declare no competing financial interests.

\section{Ethics approval and consent to participate}


bioRxiv preprint doi: https://doi.org/10.1101/244657; this version posted January 8, 2018. The copyright holder for this preprint (which was not certified by peer review) is the author/funder, who has granted bioRxiv a license to display the preprint in perpetuity. It is made available under aCC-BY-NC-ND 4.0 International license.

Not applicable.

\section{Consent for publication}

$680 \quad$ Not applicable.

\section{Acknowledgements}

682

Not applicable. 


\section{Figure Legends}

685 Figure 1. Geographic distribution of all sampling sites in Honghu lake. L: Lake; P: Pond; R:

686 River. (A) Definitions of the various sampling strategies. (B) Locations of sampling sites and the

687 distribution of the sampling medium collected at each site.

688

689

Figure 2. Taxonomic composition and relative abundances of microbial taxa in water and

sediment samples. (A) Taxonomic composition of each sample at the phylum level. 'Other'

represents all phyla not in the top 13 phyla. (B) Bar plot highlighting differences between water and

sediment samples at phylum level. ** represents $p$-values $<0.01$ and determined by a student $t$-test.

693

(C) Bar plot highlighting differences in water samples at the phylum level between impacted and

less-impacted groups. * represents $p$-values $<0.05$ and determined by a student $t$-test. (D) Bar plot

696 less-impacted groups. * represents $p$-values $<0.05$ and determined by a student $t$-test. ** represents

$697 p$-values $<0.01$ and determined by a student $t$-test.

698

699 Figure 3. Core- and Pan-OTUs of water and sediment samples from Honghu lake. Flower plots

700 showing the number of sample-specific OTUs (in the petals) and Core-OTUs (in the center) for (A)

701 all samples, (B) all water samples, and (C) all sediment samples. OTU accumulation curves for 702 Pan-OTUs (upper) and Core-OTUs (lower) for (D) all samples, (E) all water samples, and (F) all 703 sediment samples from Honghu lake.

704

705 Figure 4. PCoA plots and UPGMA-based clustering of water and sediment microbial 706 communities. Unweighted UniFrac dissimilarity matrix scores for all samples visualized in a PCoA 707 plot to demonstrate the dissimilarity of the microbial community structure between samples by (A) 
708

709

710

711

712

713

714

715

716

717

718

719

720

721

722

723

724

725

726

727

728

729

730

731

732

733

734

735

736

sampling medium (water vs. sediment) and by (B) impacted and less-impacted groups. (C) UPGMA-based clustering tree of microbial communities using an unweighted UniFrac distance matrix. The green and pink font represent less-impacted and impacted groups, respectively. The blue and red bars mark water and sediment samples, respectively.

Figure 5. Canonical correspondence analysis plots of physicochemical properties and antibiotic data driving water and sediment microbial community structure. Physicochemical properties of (A) water samples (B) and sediment samples from Honghu lake, and antibiotic data for (C) water samples and (D) sediment samples from Honghu lake. *** represents $p$-values $<0.001$, ** represents $p$-values $<0.01$ and $*$ represents $p$-values $<0.05$.

Figure 6. Environmental drivers of microbial community composition in water and sediment samples. Pairwise comparisons of (A) water and (B) sediment physicochemical properties with taxonomic and functional composition data. Color gradient represents Pearson's Correlations Coefficients. Pairwise comparisons of (C) water and (D) sediment antibiotic concentration data with taxonomic and functional composition data. In all figures, the varying circle size represents the absolute value of the Pearson Correlation Coefficient between the two factors, the bar along the y-axis represents the value of the Pearson Correlation Coefficients, and the edge width represents the Mantel's $r$ statistic value for distance correlations and the edge color denotes the statistical significance based on 9,999 permutations. Abbreviations of physicochemical properties and antibiotics are listed in sub-section 'Physicochemical characterization and antibiotic analysis'.

Figure 7. Biomarkers analysis of water and sediment microbial communities from impacted and less-impacted (control) sites. (A) Differentially abundant taxa of water samples; (B) Cladogram showing the phylogenetic structure of the microbiota from water samples; (C) Differentially abundant taxa of sediment samples; (D) Cladogram showing the phylogenetic structure of the microbiota from sediment samples.

Figure 8. Co-occurrence network interactions of Honghu lake microbes in water and sediment 
737 samples. Network nodes represent OTUs and edges are represented as interactions between OTUs.

738 All edges represent positive, strong (Spearman's $\rho>0.8$ ), and significant ( $p$-value<0.001) correlations.

739 The size of each node is proportional to the node degree. Networks of (A) water and (B) sediment

740 samples displaying co-occurrence patterns of OTUs grouped at the phylum level. Modules were

741 identified using the WalkTrap community detection algorithm in (C) water and (D) sediment samples.

742 Water (E) and (F) sediment networks investigating the effect of long-term agricultural activities

743 wherein each node was colored as function of its relative abundance in impacted and less-impacted

744 sites. 


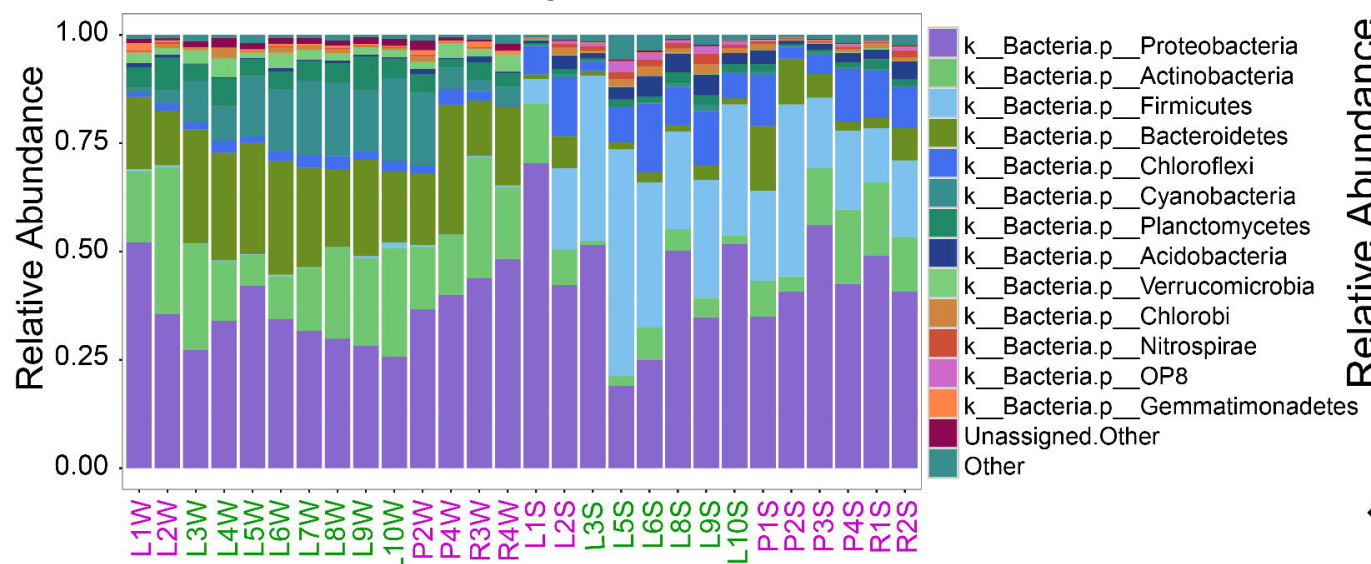

(C)
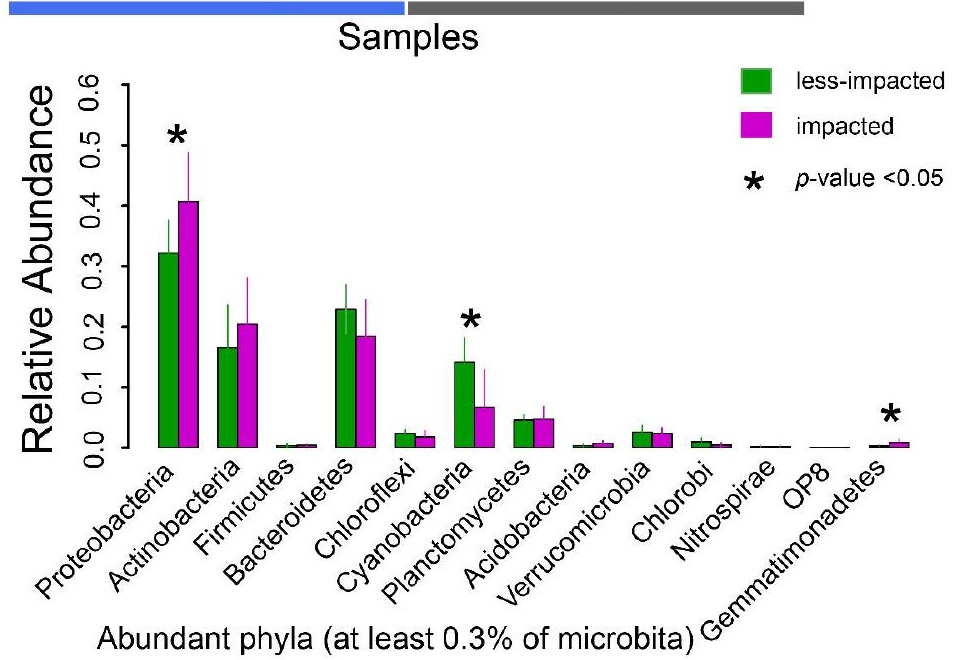

Abundant phyla (at least $0.3 \%$ of microbita)

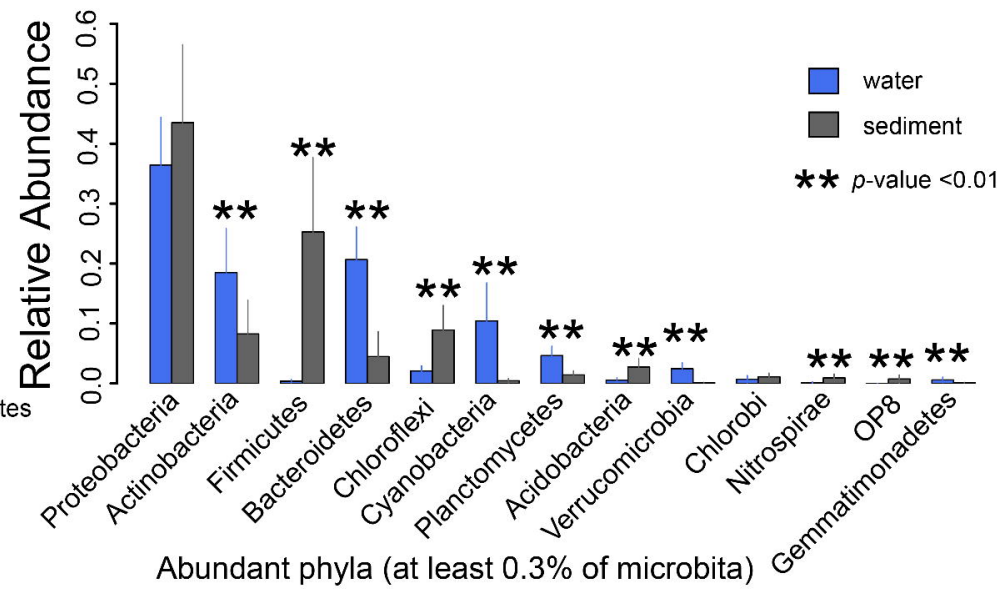

(D)

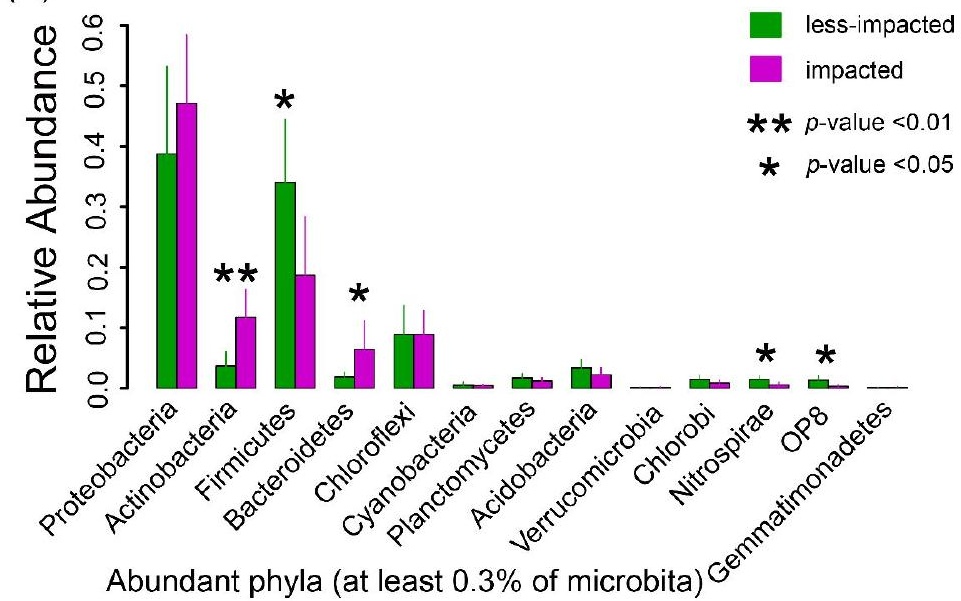




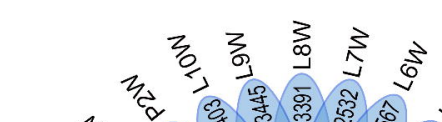

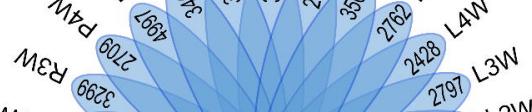

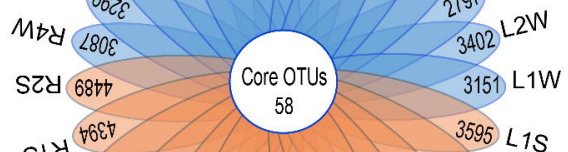

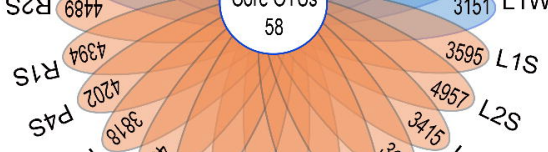

20 की के की

(D)

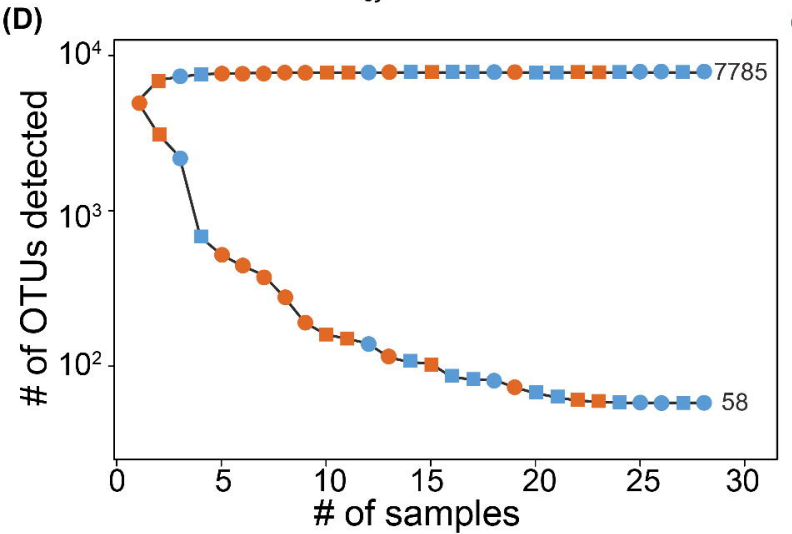

(B)

$\begin{array}{lll}3 & \text { (C) }\end{array}$

(E)
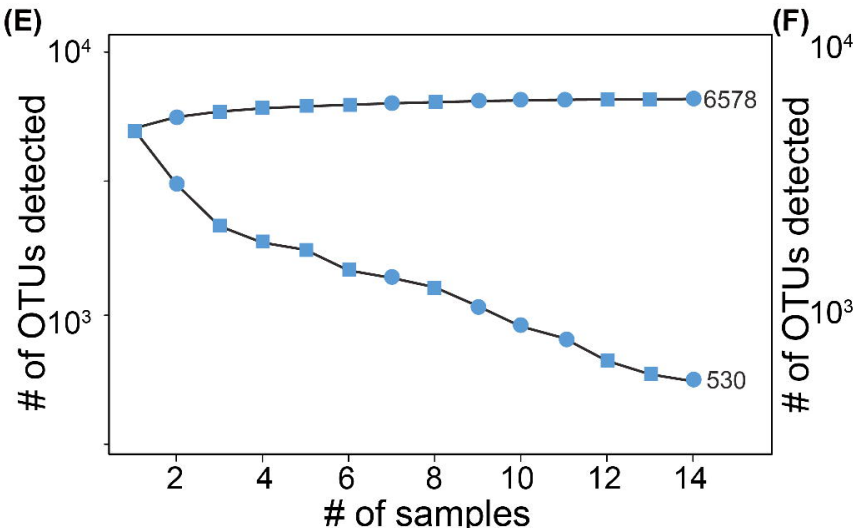

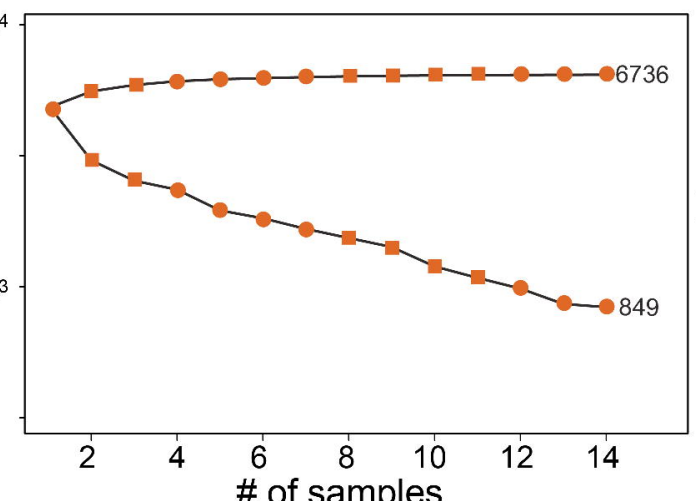

Legend

$C_{\text {Core-OTUs }}^{\text {Pan-OTUS }}$

O impacted

$\square$ less-impacted

water sample

sediment sample

\# of samples 
(A)

(C),

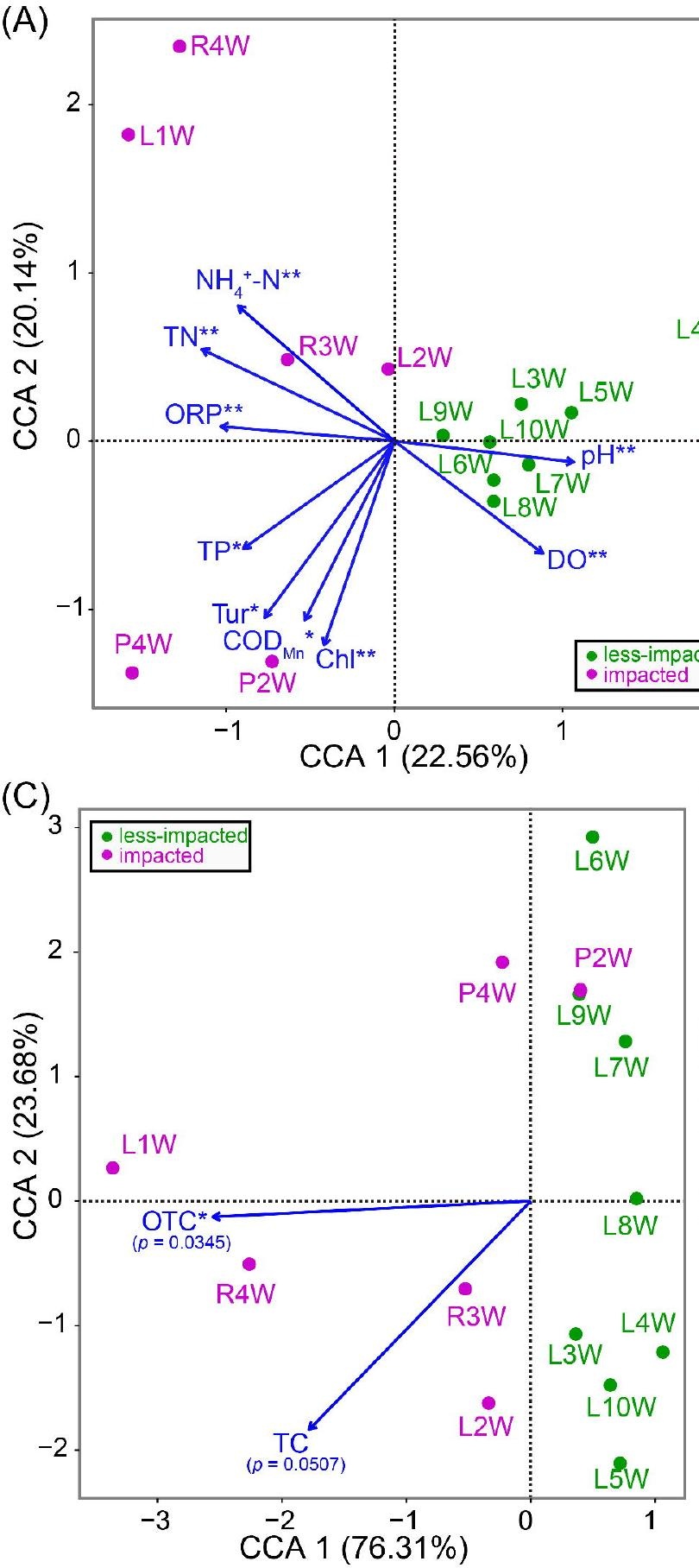

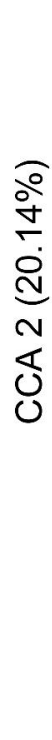

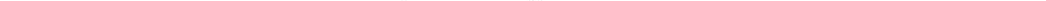

(B)

- R2S

(D)

2
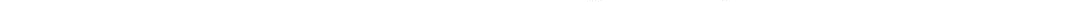

CCA $1(59.70 \%)$ 


\section{$\frac{(\mathrm{A})}{\text { Mantel }}$}

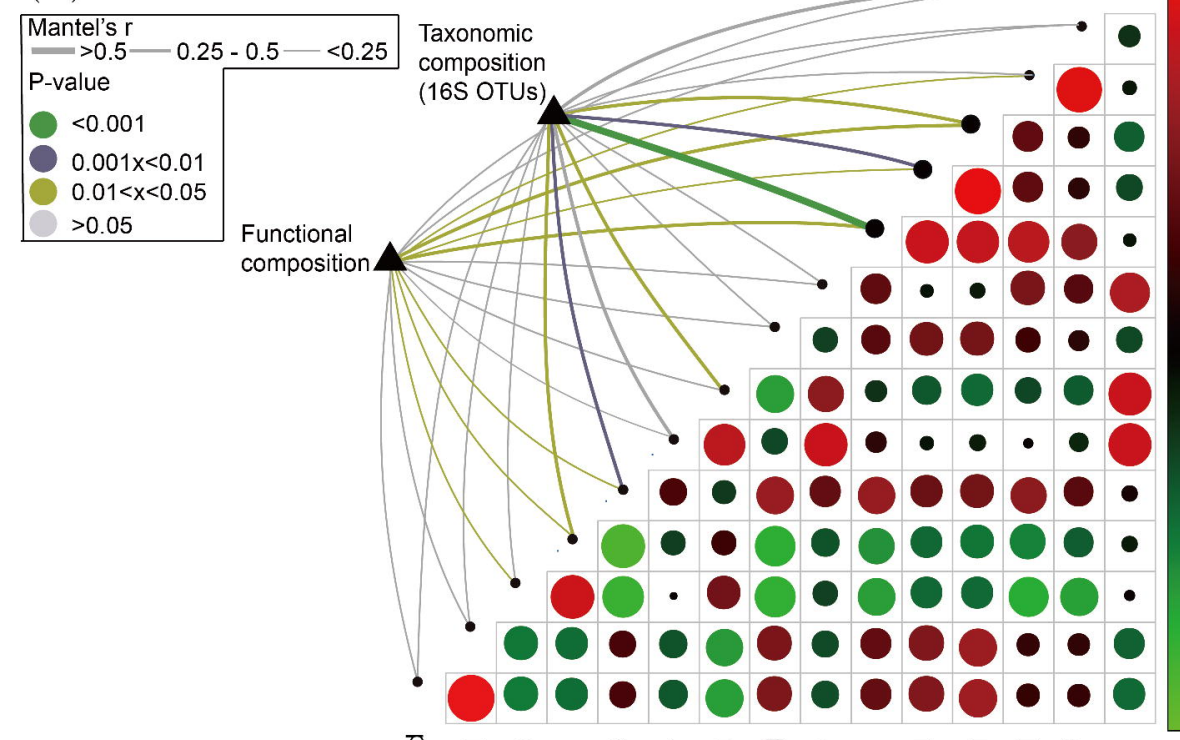

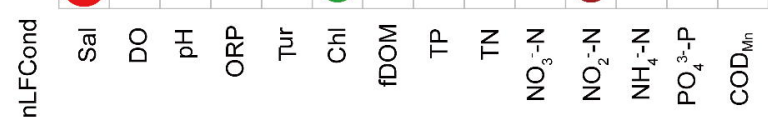

(C)

$\underset{\text { Mantel's } r}{\longrightarrow}>0.5-0.25-0.5-<0.25$ $P$-value $<0.001$ $0.001 x<0.01$ $0.01<x<0.05$ $0.01<x<4$
$>0.05$

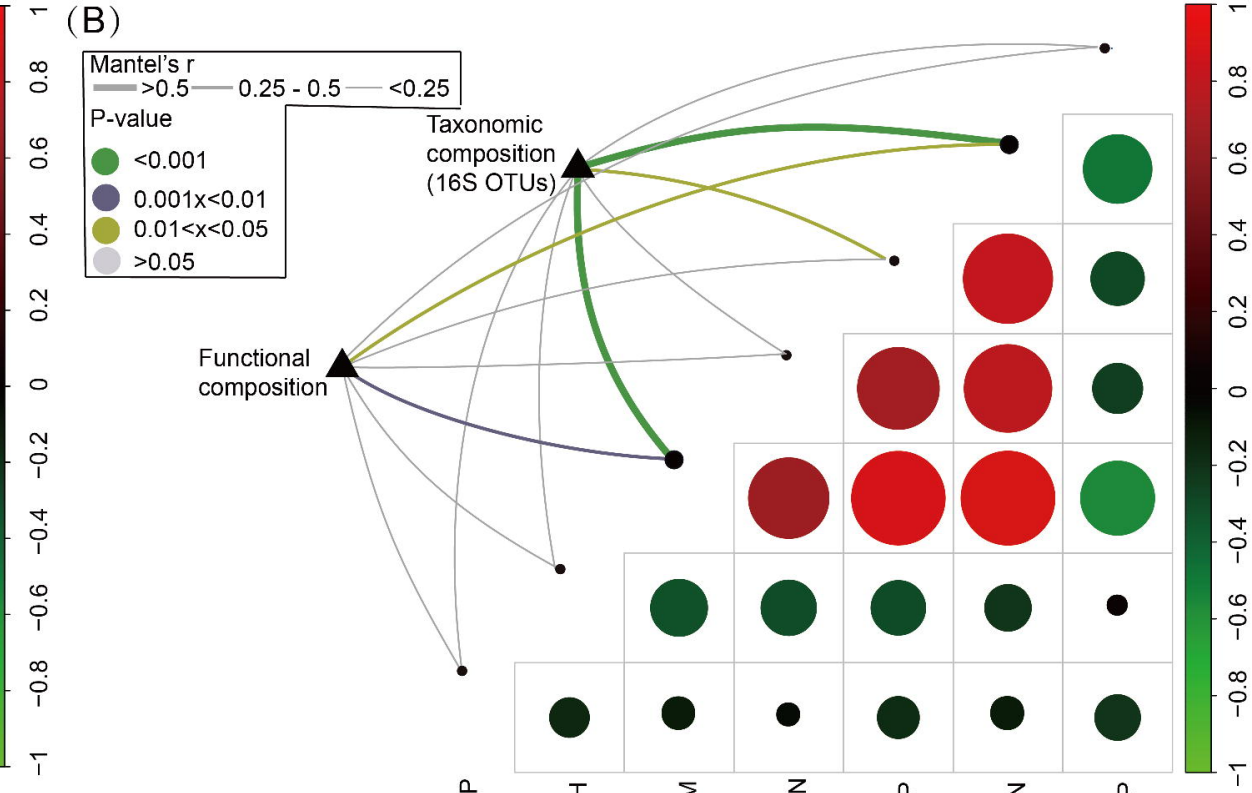

(D)

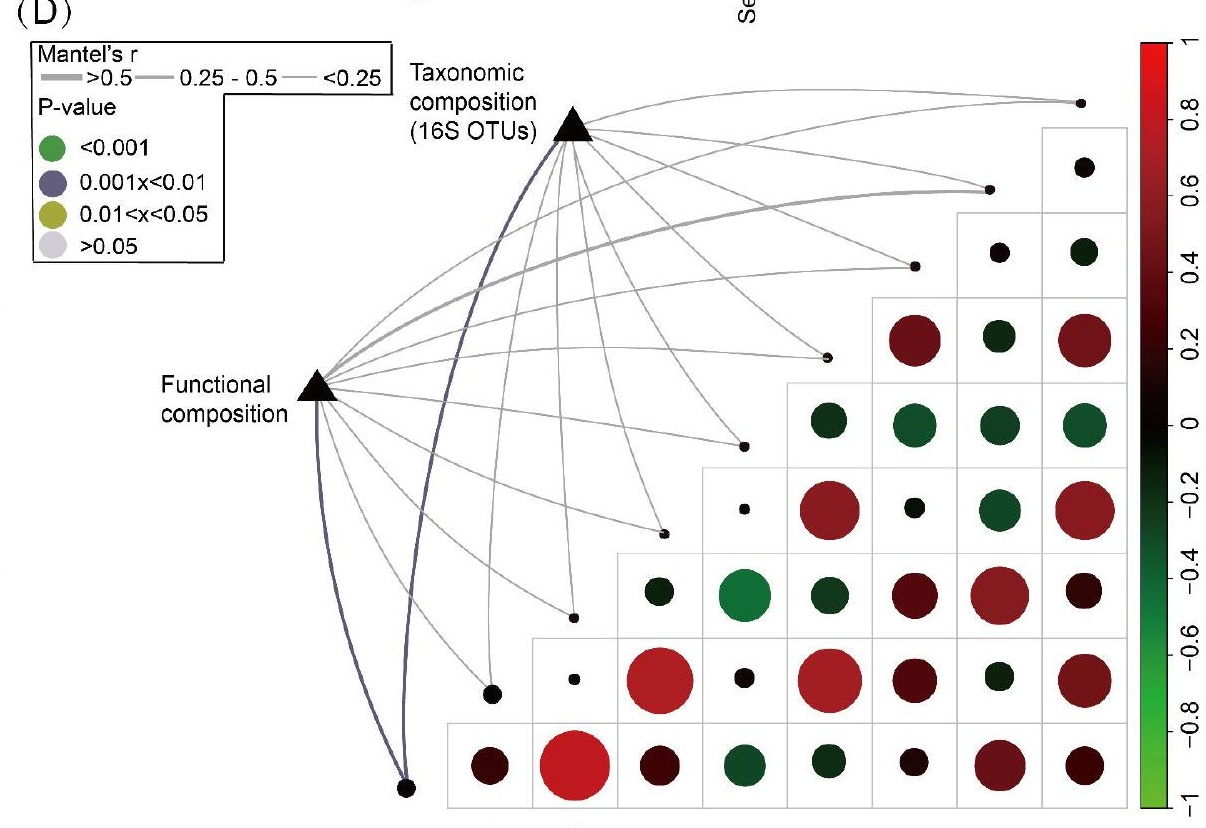

$\begin{array}{ccccccccc}0 & 0 & 0 & N & \frac{\alpha}{i} & \sum_{0}^{0} & \vec{J} & \frac{a}{U} & \sum_{0}^{N}\end{array}$ 


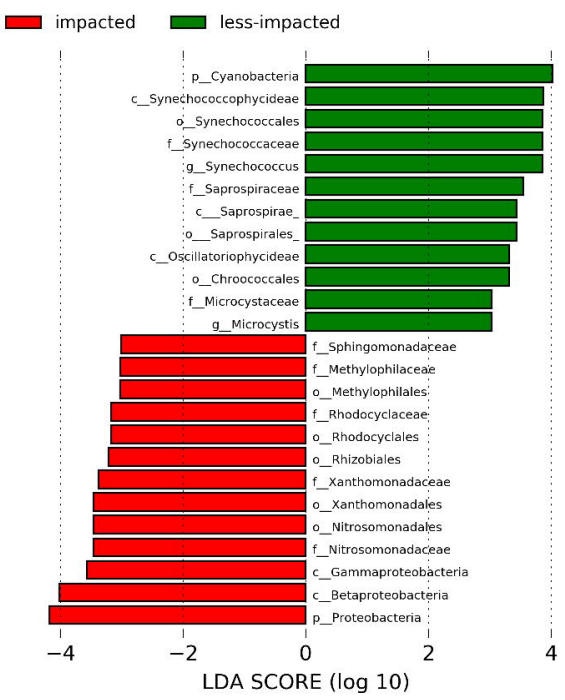

(C) Differently abundant taxa of sediment samples

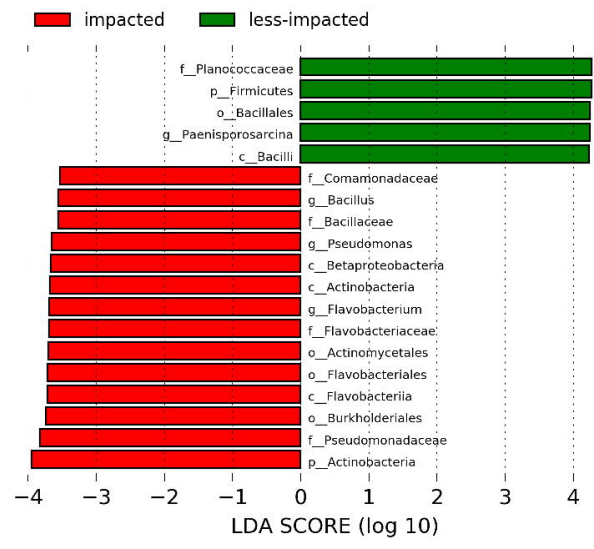

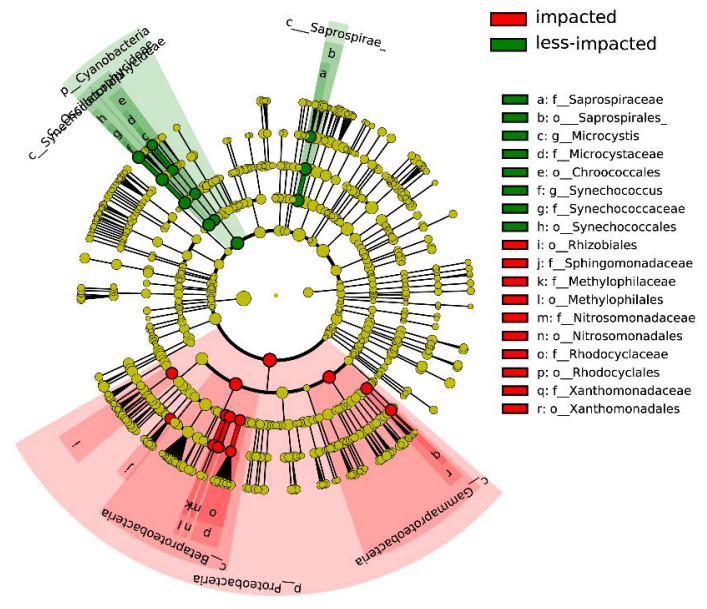

(D) Taxa cladogram of water samples

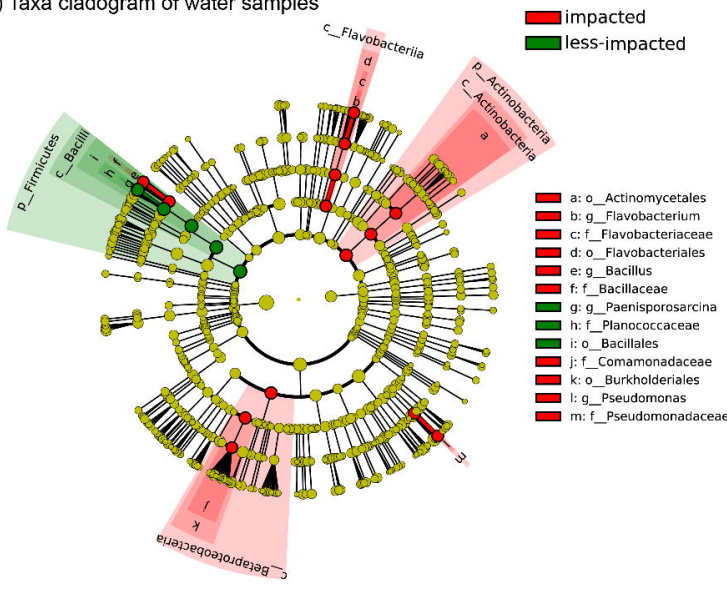


